Revue internationale P.M.E.

Économie et gestion de la petite et moyenne entreprise

\title{
Internationalisation et pratiques de gestion des ressources humaines en PME
}

\section{Mohamed Bayad}

Volume 10, numéro 3-4, 1997

URI : https://id.erudit.org/iderudit/1009030ar

DOI : https://doi.org/10.7202/1009030ar

Aller au sommaire du numéro

Éditeur(s)

Presses de l’Université du Québec

ISSN

0776-5436 (imprimé)

1918-9699 (numérique)

Découvrir la revue

Citer cet article

Bayad, M. (1997). Internationalisation et pratiques de gestion des ressources humaines en PME. Revue internationale P.M.E., 10(3-4), 57-80.

https://doi.org/10.7202/1009030ar

\section{Résumé de l'article}

L'objectif de cet article est de présenter quelques résultats d'un travail de recherche sur les orientations et pratiques de gestion des ressources humaines selon l'importance et la nature de l'engagement de la petite et moyenne entreprise industrielle sur les marchés étrangers. Dans une perspective encore peu développée, sicen'est dans la dimension management international des ressources humaines de la grande entreprise, cette investigation des relations entre GRH et degré d'engagement international est menée à partir d'un échantillon de 299 PME industrielles de la région lorraine. Les résultats démontrent que le degré de développement international n'exerce pas d'effets lourds sur les principales orientations de GRH des PMI de notre échantillon. 


\title{
Internationalisation et pratiques de gestion des ressources humaines en PME
}

\author{
Mohamed BAYAD \\ Université de Strasbourg III \\ et Université de Nancy II
}

MOTS CLÉS

\section{Stratégie - International - GRH - Exportation - PME - Dirigeant - Performance}

\begin{abstract}
RÉSUMÉ
L'objectif de cet article est de présenter quelques résultats d'un travail de recherche sur les orientations et pratiques de gestion des ressources humaines selon l'importance et la nature de l'engagement de la petite et moyenne entreprise industrielle sur les marchés étrangers. Dans une perspective encore peu développée, si ce n'est dans la dimension management international des ressources humaines de la grande entreprise, cette investigation des relations entre GRH et degré d'engagement international est menée à partir d'un échantillon de 299 PME industrielles de la région lorraine. Les résultats démontrent que le degré de développement international n'exerce pas d'effets lourds sur les principales orientations de GRH des PMI de notre échantillon.
\end{abstract}

\section{L'AUTEUR}

Mohamed Bayad est maître de conférences en sciences de gestion à l'ESM - Université de Metz. Il est aussi responsable de DESS « Entrepreneuriat et développement des PME " ainsi que de l'équipe de recherche "Ressources humaines et organisation " du CEREMO. Ses thèmes de recherche sont l'interface entre management stratégique et la GRH en contexte entrepreneurial/ PME et l'interculture.

Adresse : 3, Place Edouard-Branly 57070 Metz, France. bayad @esm.univ-metz.fr 


\section{ABSTRACT}

The goal of this paper is to present some results from a research work on the human resources management orientations and practices according to the importance and the nature of the commitment of the industrial SMB on the foreign markets.

In a few developed perspective, apart from in the dimension of the international management of the human resources within big firms, this investigation related to the links between the HRM and the international commitment level is conducted from a sample of 299 industrial SMB located in Lorraine (region in the East of France). The results obtained suggest that the international development degree do not exert any heavy effects on the main orientations of HRM of the SMB pertaining to our sample.

\section{RESUMEN}

El objetivo de este articulo es de presentar algunos resultados de un trabajo de busqueda sobre las orientaciones y prácticas de gestión de recursos humanos según la importáncia y la naturaleza del empeño de la pequeña y mediana empresa industrial en los mercados extranjeros.

En una perspectiva poco desarrollada aún, salvo en la dimensión del manejamiento internacional de recursos humanos de la gran empresa, esta investigación de las relaciones entre la gestión de recursos humanos y el grado de empeño internacional es llevada a partir de una muestra de 299 pequeñas y medianas empresas industriales de la región lorena. Los resultados obtenidos sugieren que el grado de desarrollo internacional no ejerce efectos pesados sobre las principales orientaciónes de la gestión de recursos humanos de las pequeñas y medianas industrias de nuestra muestra.

\section{Introduction}

Face à la mondialisation des économies, l'engagement sur les marchés étrangers apparaît aujourd'hui comme un défi de taille pour les entreprises (Perlmutter et Heenan, 1979; Ohmae, 1985 ; Chandler, 1986 ; Porter, 1986 ; Urban, 1993 ; Joffre, 1994). L'internationalisation prend ainsi une dimension essentielle pour le développement et les choix stratégiques des entreprises de toutes tailles.

L'évolution des exportations, expression première de l'internationalisation, met en relief le dynamisme particulier des petites et moyennes entreprises industrielles (PME / PMI) ${ }^{1}$ sur les marchés étrangers durant les années 1980. Même s'il convient de garder à l'esprit que toutes les PMI ne sont pas faites pour exporter

1. En 1990, un quart des exportations françaises a été réalisé par des PMI. Par ailleurs, entre 1984 et 1989 , les exportations directes des PMI se sont accrues de $59 \%$ contre $36 \%$ pour les grandes entreprises exportatrices (d'après L'entreprise/INSEE, numéro spécial, 1992). 
(De Leersnyder, 1980), le développement international est un prolongement naturel des stratégies de spécialisation de ces entreprises et, parallèlement, il constitue une option à leurs stratégies de diversification «nationale » (Bilkey, 1978; Saporta, 1986 ; Namiki, 1988 ; Chaganti, Chaganti et Mahajan, 1989 ; Baird, Lyles et Orris, 1994 ). Certaines PME, à partir de motivations semblables à celles des grandes entreprises (opportunités de croissance à l'échelle mondiale) mais avec des stratégies génériques différentes (micromarchés versus marchés de masse), n'hésitent pas à franchir le pas de l'investissement direct tant en matière de commercialisation que de filiales de production (Namiki, 1988).

La compétitivité, dans un espace concurrentiel mondial riche en diversités culturelles et en disparités géographiques, sollicite l'ensemble des ressources et des compétences de l'entreprise internationale. Le rôle des ressources humaines dans la création d'un avantage concurrentiel à l'international ne semble avoir soulevé que peu d'intérêt tant au regard de la recherche appliquée en management stratégique et en gestion des ressources humaines qu'au regard des pratiques managériales des entreprises internationales. Comme le souligne Adler (1994), ce constat est d'autant plus inquiétant que le personnel le plus impliqué dans les stratégies d'internationalisation est, lui-même, souvent ignoré :

Dans une foule de domaines, on fait de plus en plus appel à des stratégies qui, il y a dix ou vingt ans, nous étaient inconnues ou nous paraissaient sans fondement. En matière de gestion du personnel international, pourtant, nombreuses sont encore les entreprises qui agissent comme si rien n'avait bougé ni dans leur structure interne, ni dans leur organisation ni dans le contexte économique et technologique où elles évoluent.

Parmi les quelques recherches sur la relation entre gestion des ressources humaines et internationalisation, la PME internationale est généralement absente pour des raisons souvent évoquées de masse critique de personnel à l'international ou encore de «politiques de GRH rarement explicitées».

Cependant, même les PME locales ont à faire face à l'international à travers l'unification progressive du marché mondial (le GATT : Accord général sur les tarifs douaniers et le commerce) et l'apparition de blocs commerciaux (marché européen, Association nord-américaine de libre-échange, etc.). Cette ouverture inéluctable des frontières n'est pas sans avoir des incidences sur les modes de gestion des ressources humaines des entreprises nationales. Rappelons, avec Hermel (1991), que :

[...] si «l'étranger » peut arriver directement aux portes de la plus petite entreprise régionale, cela signifie que les effets et les impacts de ce phénomène sont possibles (directement ou de façon diffuse) sur toutes les fonctions, y compris sur la gestion des ressources humaines et sur le management. 
Dans cette perspective d'approche de l'ouverture internationale comme source d'effets multiples sur l'entreprise, l'objectif de cet article est de présenter les résultats d'un travail de recherche sur les pratiques de GRH selon l'ampleur et la nature de l'engagement de la petite et moyenne entreprise industrielle sur les marchés étrangers.

Après avoir présenté le cadre de l'étude, la problématique de recherche et les aspects méthodologiques, nous axerons cet article sur les principaux résultats ainsi que sur les implications managériales qui en découlent pour le dirigeant de PME et, plus généralement, sur le management des ressources humaines face à l'international.

\section{Cadre de l'étude}

\subsection{Décision et performance à l'export de la PME : les dimensions explorées}

La prise en compte des enjeux pour les PME des nouvelles conditions de l'échange international n'a pas été sans soulever un grand nombre de questions (Orsini, 1985). Celles-ci ont donné lieu, durant ces dernières années, à de nombreuses études cherchant à comprendre ce choix d'orientation vers les marchés étrangers (Bilkey et Tesar, 1977 ; Cavusgil et Nevin, 1981 ; Ditchl et al., 1984 ; Roux, 1986 ; Baird, Lyles et Orris, 1994). Ces recherches portent généralement sur les relations entre comportement à l'exportation et caractéristiques de la PME. Deux aspects sont particulièrement étudiés : l'aspect décisionnel (facteurs d'engagement et processus de développement à l'international) et l'aspect performance (facteurs de succès ou d'échec à l'international).

Dans une perspective classique de management stratégique, décision et performance de l'engagement international apparaissent relever de facteurs externes et internes à l'entreprise (Aaby et Slater, 1989, Roux, 1991; Bijmolt et Zwart, 1994). Si les facteurs externes, relatifs aux différents environnements de l'entreprise, sont généralement considérés comme des contraintes ${ }^{2}$ (menaces / opportunités), les développements les plus récents s'attachent aux facteurs internes (forces / faiblesses) de l'internationalisation des PME (Joyal, Julien, Deshaies et Ramangalahy, 1996 ;

2. S'il est vrai que les environnements micro (secteur d'activités, intensité concurrentielle, changement technologique, etc.) et macro (politiques gouvernementales, réglementation, taux de change, etc.) offrent peu de marge de manœuvre à la PME, il existe un environnement intermédiaire que l'on peut qualifier de méso (district industriel et parc technologique associés à un espace géographique limité) sur lequel la PME est un acteur à part entière. Le concept de " glocalisation » proposé par Johanisson (1994) pour analyser le processus d'internationalisation de la PME illustre parfaitement l'accès à un marché étranger par la combinaison d'opérations commerciales globales et de coopérations locales entre PME. 
Bijmolt et Zwart, 1994 ; Baird, Lyles et Orris, 1994 ; Ogbuehi et Longfellow, 1994). Notons que des auteurs comme Cavusgil (1984) avaient, depuis longtemps déjà, appelé à une accentuation de la recherche sur les déterminants internes du comportement à l'exportation des entreprises :

External factors, such as exchange rate, level of domestic and foreign demand, relative rates of price level increases, and commercial policy proved to be poor predictors of a firm's export behavior [...] In order to understand variations among firms with respect to the existence and the extent of export activity one must explore some crucial factors within the firm and its management.

Un relatif consensus permet de dresser une typologie en quatre grandes catégories des déterminants internes du comportement à l'exportation de la PME (Bilkey, 1978; Miesenbock, 1988; Aaby et Slater, 1989; Roux, 1991).

- Les caractéristiques de l'entreprise : les variables explicatives les plus étudiées sont la taille, le degré de maturité, l'expérience internationale et le type d'activité de la PME.

- Les compétences de l'entreprise : parmi l'ensemble des variables descriptives des savoir-faire de l'entreprise, l'accent est surtout mis sur la maîtrise technologique, la capacité d'innovation (recherche et développement) et l'expertise de l'organisation export (information, planification, contrôle, communication, etc.).

- La stratégie d'internationalisation : partie intégrante de la stratégie globale de l'entreprise, elle est surtout abordée sous l'angle de la stratégie marketing (sélection des marchés, prix, publicité, canaux de distribution).

- Les caractéristiques du dirigeant : acteur incontournable en PME, le rôle du dirigeant dans l'ouverture internationale de l'entreprise a été analysé sous le prisme de ses caractéristiques individuelles (formation, expérience, etc.), sa personnalité, ses motivations, ses valeurs, son attitude à l'égard du risque, ses objectifs et sa vision stratégique internationale.

Les recherches empiriques sur ces quatre catégories de facteurs internes font tout d'abord ressortir que les attitudes et attentes du dirigeant (objectifs, motivation, prise de risque, capacité de traitement de l'information et d'adaptation) en ce qui concerne l'international sont déterminantes (Ogbuehi et Longfellow, 1994). Il apparaît ensuite que les stratégies d'engagement et de développement international ne sont pas sans incidences sur les comportements de gestion et les différentes fonctions de l'entreprise (Orsini, 1985 ; Léo, Monnoyer-Longe et Philippe 1990; Bijmolt et Zwart, 1994). Ainsi, parallèlement à une modification de la vision stratégique internationale du dirigeant (apprentissage), les domaines de l'organisation export (Samiee et Walters, 1990) et du marketing en particulier (Koe et Robicheaux, 1988) sont les plus concernés pour concrétiser la volonté d'ouverture et les efforts 
engagés à chaque étape du processus de développement international de la PME. Soulignons, enfin, que le succès sur le plan international nécessite que la décision d'exporter relève d'une véritable volonté stratégique et soit, de ce fait, en parfaite cohérence avec la stratégie globale de l'entreprise.

\subsection{La GRH et l'international: la dimension oubliée}

À la lumière des travaux de recherche sur le développement international de la PME, la fonction ressources humaines $(\mathrm{RH})$, ou tout au moins les activités d'acquisition, de stimulation et de développement des RH n'apparaissent pas, a priori, comme des éléments importants d'évaluation dans la démarche stratégique à l'international du dirigeant. Ce constat n'est d'ailleurs pas spécifique à la PME et la dimension humaine des organisations est loin d'être pleinement intégrée dans le management stratégique des grands groupes multinationaux (Dowling et Schuler, 1990; Adler, 1994).

De manière très visible dans les recherches en GRH, cette perspective est remise en cause avec la reconnaissance de la dimension stratégique des ressources humaines dans le développement de l'entreprise (Fombrun, DeVanna et Tichy, 1982; Baird et Meshoulam, 1988).

La GRH considérée dans sa dimension stratégique (gestion stratégique des ressources humaines) est un vecteur de première importance pour la réussite de l'entreprise et soulève de fait un double défi managérial de cohérence externe et de cohérence interne :

Creating effective strategic human resource practices requires the management of two fits simultaneously, external and internal. The external fit involves the fit between HRM (Human Resource Management) activities and the organization context at each major developmental stage (e.g. life cycle phase) of the organization; the internal fit requires that the components of human resource management must fit with and support each other. (Baird et Meshoulam, 1988)

Les travaux concernant la cohérence externe dominent par leur nombre le champ de la GSRH. Ils sont néanmoins fortement remis en cause pour leur caractère déterministe ; les RH s'ajustent aux choix stratégiques et à l'évolution organisationnelle. A contrario, pour les recherches sur la cohérence interne, la GRH n'obéit pas mécaniquement à la stratégie d'entreprise, mais elle est une fonction organique ; les RH, comme les autres ressources de l'organisation, sont génératrices d'une compétence distinctive durable.

L'introduction de cette dimension stratégique des RH dans un contexte international ne pouvait que donner lieu à la création de modèles intégrés de gestion stratégique internationale de ressources humaines (GSIRH) comme celui de Schuler, Dowling et De Cieri (1993). En s'inscrivant spécifiquement dans l'espace 
des grands groupes multinationaux, la GSIRH trouve, selon ces auteurs, ses fondements dans la reconnaissance :

a) that human resource management at any level is important to strategy implementation; b) that major strategic components of multinational enterprises (MNE) have a major influence on international management issues, functions, policies and practices; $c$ ) that many of these characteristics of SIHRM (GSIRH) can influence the attainment of concerns and goals of MNEs; d) that there are a wide variety of factors that make the relationship between MNEs and SIHRM complex, thereby making the study of SIHRM challenging as well as important.

Dans le cadre de ce type de modèle, la GRH considérée dans sa dimension internationale concerne, plus particulièrement, la gestion des « expatriés », celle des «cadres sans frontières » des firmes multinationales (Audibet et Parlant, 1990), «l'interculturel » ou la reconnaissance des spécificités culturelles locales en fonction des différentes étapes du développement international allant de la phase nationale (marché domestique) à la phase de globalisation (marché transnational) [Adler, 1994; Vidal, 1991]. D'un intérêt indéniable, ces recherches n'ont malheureusement donné lieu qu'à un faible nombre d'investigations empiriques.

Quoi qu'il en soit, la PME est absente de ces recherches qui se focalisent en majorité sur la multinationale. Cependant, les RH et leur mode de gestion devraient jouer un rôle déterminant dans les stratégies de développement international de la PME. De ce point de vue, quelques études mettent en relief le poids de certaines pratiques de GRH (recrutement, rémunérations et formes de motivations) sur les performances à l'export (Gomez-Meija, 1988) ainsi que les coûts d'ajustement assumés par le dirigeant et le personnel dans l'engagement international (Wright, 1993). Ces quelques études sont à la base de notre problématique.

\section{GRH, international et PME: problématique et méthodologie}

\subsection{Les questions de l'étude}

En reprenant la problématique de la relation entre GRH et développement international (Hermel, 1991 ; Chase, 1984 ; Gomez-Meija, 1988 ; Wright, 1993), cette étude tente d'évaluer empiriquement l'impact de l'internationalisation sur les orientations et les pratiques de GRH de la PME industrielle tout en tenant compte, d'une part, de certains paramètres qui ne sont pas sans incidences sur la GRH de la PME (Bayad, 1991 ; Bayad et Herrmann, 1992) et, d'autre part, des effets possibles sur la performance à l'exportation. 


\section{GRAPHIQUE 1}

\section{Problématique et questions de l'étude}

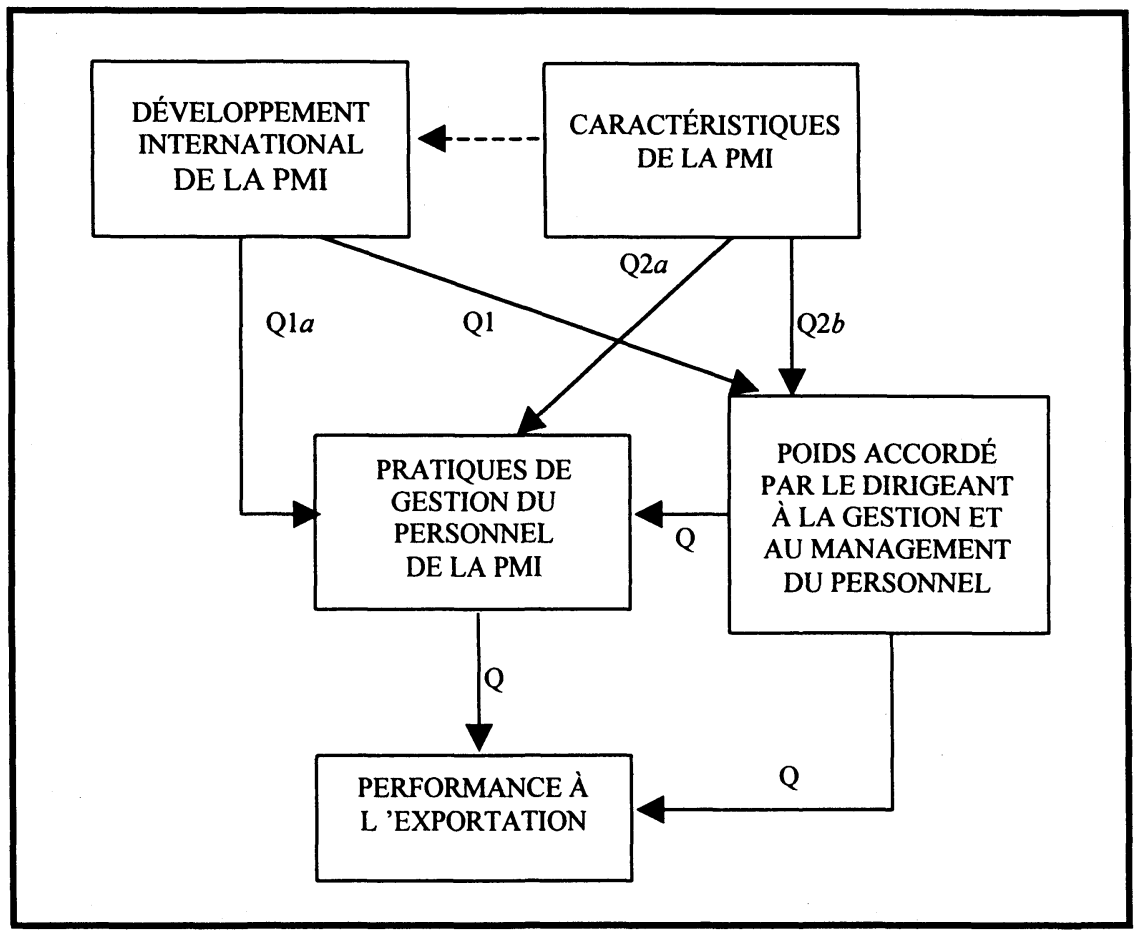

De manière plus précise, notre problématique de recherche (graphique 1) repose sur la série de questions suivantes :

- Q1: Le développement international de la PMI exerce-t-il une influence :

Q1 $a$ : sur la place accordée aux différents axes de pratiques de GRH?

Q1b: sur le degré d'importance accordé par le dirigeant à la gestion et au management du personnel?

- Q2: Les caractéristiques structurelles de base de la PMI ont-elles une incidence :

Q2a: sur la place accordée aux différents axes de pratiques de GRH?

$\mathrm{Q} 2 b$ : sur le degré d'importance accordé par le dirigeant à la gestion et au management du personnel?

- Q3 : Le poids accordé par le dirigeant à la gestion et au management du personnel détermine-t-il les orientations de GRH de la PMI?

- Q4: La place accordée aux différents axes de pratiques de GRH a-t-elle un effet sur la performance à l'exportation de la PMI ?

- Q5: Le poids accordé par le dirigeant à la gestion et au management du personnel a-t-il un impact sur la performance à l'exportation de la PMI? 


\subsection{L'échantillon de travail}

L'investigation est menée à partir d'un échantillon de 299 PME industrielles issu de la troisième vague d'enquête (1991) du Panel PMI lorraines portant sur l'exercice $1990^{3}$. Les entreprises concernées relèvent de l'industrie manufacturière ${ }^{4}$ et employent de 6 à 500 salariés ( $88 \%$ des entreprises de l'échantillon ont moins de 200 salariés et $50 \%$, moins de 50 salariés), pour les quatre départements de la région lorraine. Relativement à l'objet d'étude et aux contraintes d'échantillonnage qui s'y rattachent (Malekzadeh et Nahavandi, 1985 ; Roux, 1986), la Lorraine, tant par sa position géographique que par sa tradition exportatrice, est une région appropriée pour l'approche des phénomènes d'ouverture internationale. À titre d'illustration, deux PMI sur trois $(66,56 \%)$ de l'échantillon de travail ont exporté en 1990. Elles ont exporté en moyenne environ $14 \%$ de leur chiffre d'affaires, ces exportations pèsent pour près de quatre milliards de francs. Parmi celles n'ayant pas exporté en 1990, $9 \%$ déclarent envisager l'adoption d'une politique d'exportation délibérée et systématique avant l'ouverture du Grand Marché européen de 1993.

\subsection{Opérationalisation et mesures}

Les informations utilisées pour l'étude seront d'abord présentées selon le degré d'ouverture internationale et les caractéristiques de la PMI; ensuite, selon les pratiques de GRH et la perception du dirigeant.

\section{Les facteurs d'influence}

L'un des principaux objectifs de cette étude est de tester les effets de l'internationalisation sur les pratiques de GRH de la PME industrielle. L'internationalisation de la PMI sera évaluée à partir de son degré d'engagement à l'exportation. Ce dernier peut s'entendre de différentes manières. Nous avons choisi de retenir quatre dimensions principales permettant de conserver dans l'analyse toutes les PMI de l'échantillon et ainsi bénéficier d'une dimension comparative générale (exportateur et non-exportateur).

3. Cette enquête a été menée annuellement par l'Institut commercial de Nancy (Groupe ICN) avec le soutien financier du Conseil régional de Lorraine depuis 1988 à 1994. L'objectif de ce programme de recherche est d'étudier et de comprendre, grâce notamment à la dimension longitudinale, les comportements et pratiques de gestion des PMI industrielles en recueillant des données empiriques au cours d'entretiens directifs (questionnaire) avec les responsables des entreprises du panel ( $c f$. Bayad et Herrmann, 1992).

4. Industries de biens intermédiaires, de biens d'équipements, de biens de consommation, et quelques entreprises de l'industrie agroalimentaire (IAA). L'échantillon de travail est représentatif de cette structure sectorielle pour la région de la Lorraine. 
- La fréquence d'exportation de l'entreprise (pas du tout, occasionnellement ou régulièrement) au cours des cinq années précédant 1990 permet d'apprécier le niveau d'engagement à l'exportation (Roux, 1986; Czinkota et Ursic, 1991).

- L'expérience à l'exportation de l'entreprise, définie par rapport aux classes d'ancienneté à partir de l'année de la première exportation, est une évaluation de l'expérience acquise ou non sur les marchés étrangers (Reffait et Roux, 1981).

- Le risque à l'exportation de l'entreprise est évalué à partir d'un classement des PMI de l'échantillon selon la proximité géographique des marchés, le nombre de pays clients et la grandeur du chiffre d'affaires (CA) à l'exportation (Bilkey et Tesar, 1977 ; Cavusgil et Nevin, 1981); par exemple, en classant à part les «Franco-Françaises », les « Régionales » sont des PMI dont la totalité du CA export est réalisée au Luxembourg, en Belgique ou en ex-RFA.

- L'organisation « export ", approchée par la fréquence de recours ou non à du personnel pour l'activité à l'exportation (jamais, à temps partiel ou à temps plein) consolidée par la présence ou non de personnes possédant une formation spécifique en commerce international, est un indicateur du degré d'implication de l'entreprise sur les marchés étrangers (Reffait et Roux, 1981 ; Samiee et Walters, 1990; Léo, 1993).

Enfin, les autres facteurs exogènes ( $c f$. graphique1) sont les caractéristiques structurelles de base de la PMI. Il s'agit de la taille (tranches d'effectifs salariés), de la maturité (tranches d'âge depuis la création) et du secteur d'activités de l'entreprise (niveau 15 de la Nomenclature d'activités et de produits). L'introduction de ces paramètres dans l'analyse provient de la nécessité de prendre en compte les effets «taille», «secteur » et "stade de développement » de la PMI, qui ont la particularité d'être sous-jacents, voire explicatifs, tant au regard du comportement à l'exportation (Reffait et Roux, 1981 ; Roux, 1986 ; Samiee et Walters, 1990 ; Léo, 1993 ; Czinkota et Ursic, 1991) qu'à celui des pratiques de GRH (Mahé de Boislandelle, 1988 ; Benoît et Rousseau, 1990 ; Garand et Fabi, 1991 ; Bayad, 1991).

\section{Les variables endogènes}

L'évaluation des pratiques de GRH de la PME a été réalisée à partir des réponses aux questions sur le degré d'importance accordé par l'entreprise à différents domaines administratifs et politiques de gestion du personnel. La formulation de ces questions est inspirée des travaux sur la GRH dans les PME de Mahé De Boislandelle (1988). L'état des pratiques de gestion du personnel de la PME est ainsi saisi par huit énoncés sur une échelle de un (pas du tout) à quatre (très important), dont certains font référence à l'administration du personnel (tenue des fichiers et 
registres du personnel, gestion de la paie, calcul des salaires, etc.) et d'autres, aux politiques de personnel (politique de rémunération, définition et organisation du travail, amélioration des conditions de travail et relations sociales, gestion du potentiel humain et formation, gestion de l'emploi, caractère prévisionnel de la gestion du personnel).

Afin de dégager, dans un cadre multivarié, les orientations synthétiques des pratiques de GRH des PMI de notre échantillon, nous avons effectué une analyse factorielle sur ces points. Celle-ci fait ressortir deux dimensions fortes qui restituent $58 \%$ de l'information sur les pratiques de gestion du personnel (graphique 2).

GRAPHIQUE 2

Principaux résultats de l'analyse factorielle (Rotation VARIMAX)

\begin{tabular}{|c|c|c|}
\hline POINTS (majeurs) & Contributions & INTERPRÉTATION \\
\hline \multirow{4}{*}{$\begin{array}{l}\text { Gestion de l'emploi } \\
\text { Gestion du potentiel humain } \\
\text { Gestion prévisionnelle } \\
\text { Organisation du travail }\end{array}$} & \multirow{4}{*}{$\begin{array}{l}0,770 \\
0,765 \\
0,681 \\
0,665\end{array}$} & FACTEUR 1 \\
\hline & & Variance expliquée $=33 \%$ \\
\hline & & Politique du personnel \\
\hline & & (-) RÉACTIVITÉ PROACTIVITÉ (+) \\
\hline & & FACTEUR 2 \\
\hline Gestion de la paie & 0,891 & Variance expliquée $=25 \%$ \\
\hline Tenue des fichiers & 0,816 & \multirow[b]{2}{*}{ Administration du personnel } \\
\hline Gestion de l'emploi & 0,474 & \\
\hline Politique de rémunération & 0,422 & $(-)$ CONTRAINTE $\quad$ ENRICHIE $(+)$ \\
\hline $\begin{array}{l}\text { Qualité de la solution factori } \\
-\% \text { de variance expliquée } \\
\text { - mesure de KAISER }=0,8\end{array}$ & & \\
\hline
\end{tabular}

Le premier axe factoriel fait référence à une orientation politique du personnel que l'on peut qualifier de «proactive versus réactive » (Besseyre Des Horts, 1988 ; Mahé de Boislandelle, 1988; Bartoli, 1991). En effet, cette dimension reflète une forte opposition entre les PMI de l'échantillon qui accordent une place importante aux aspects stratégiques de la GRH, à savoir la gestion de l'emploi, la formation, la prévision et l'organisation du travail (graphique 2) et celles qui ne s'en préoccupent pas autant. Par ailleurs ${ }^{5}$, cet axe factoriel est significativement et positivement lié au degré de formalisation et de standardisation des PMI de l'échantillon. Le deuxième axe est à la base d'une opposition appréciable entre les PMI de l'échantillon qui attachent une grande importance à la gestion des salaires et à la

5. Dans le cadre d'une étude parallèle en cours, les deux dimensions factorielles de cette analyse, illustrées à l'aide de variables d'appui (profil du dirigeant, formalisation, standardisation, etc.) sont à la base d'une typologie très significative des pratiques de GRH dans les PMI. 
l'échantillon qui attachent une grande importance à la gestion des salaires et à la tenue de fichiers-registres du personnel, en connexion avec la gestion de l'emploi et la politique de rémunération, à celles qui n'accordent qu'un minimum de place à ces aspects de gestion du personnel. Cet axe est le reflet d'une orientation administration du personnel «contrainte versus enrichie». Ce second facteur est en relation étroite et positive avec la présence d'un responsable en titre du service du personnel. Ces deux dimensions synthétiques sont retenues comme les orientations principales, en termes de continuum, des pratiques de GRH des PMI de l'échantillon.

En ce qui concerne le poids accordé par le dirigeant à la gestion et au management du personnel, comparativement aux autres domaines fonctionnels de l'entreprise, nous avons retenu le degré d'importance accordée (échelle de un à quatre), par celui-ci, d'une part, à la gestion du personnel et, d'autre part, à l'organisation et à la direction des hommes. Enfin, toujours dans la dimension endogène, la performance à l'exportation est appréciée à partir du chiffre d'affaires à l'export.

\subsection{Méthode statistique}

Les réponses aux différentes questions de la problématique reposent toutes sur les tests F (de Fisher) associés aux différentes analyses de variance (procédure ANOVA) à plusieurs facteurs (et effets d'interaction). Afin de répondre à la question Q4 (graphique 1), compte tenu de la méthode statistique retenue, les deux axes factoriels relatifs aux orientations principales de GRH ont donné lieu chacun à un découpage en classes d'effectifs à peu près égaux.

\section{Résultats}

Avant de présenter les résultats qui nous intéressent, il convient de garder à l'esprit que des liaisons existent bien entre les données relatives au degré et à la nature du développement international et certaines caractéristiques de base de la PME, comme sa taille et son stade de développement économique (âge de l'entreprise). Cela confirme que le comportement commercial à l'exportation est très dépendant de facteurs structurels caractérisant l'entreprise (Reffait et Roux, 1981 ; Czinkota et Ursic, 1991). Nous reviendrons sur ce dernier point lorsque nous examinerons, plus en détail, les résultats obtenus.

\subsection{Effets du développement international sur la GRH de la PMI}

En ce qui concerne les incidences du degré d'internationalisation sur les orientations de gestion du personnel, les résultats obtenus permettent d'observer que seules l'organisation et la fréquence à l'exportation ont des effets significatifs. Ces effets sont cependant faibles et à la limite de la significativité ; respectivement à $4 \%$ et $6 \%$ et une variance expliquée de l'ordre de $4 \%$ (tableau 1). 
TABleau 1

Effets de l'internationalisation sur la GRH de la PMI

\section{DÉVELOPPEMENT INTERNATIONAL}

\begin{tabular}{|c|c|c|c|c|c|}
\hline & $\begin{array}{l}\text { Expérience } \\
\text { export }\end{array}$ & $\begin{array}{l}\text { Fréquence } \\
\text { export }\end{array}$ & $\begin{array}{l}\text { Risque } \\
\text { export }\end{array}$ & $\begin{array}{c}\text { Organisation } \\
\text { export }\end{array}$ & $\begin{array}{c}R 2^{a} \\
(\operatorname{Pr}>\mathrm{F})\end{array}$ \\
\hline \multicolumn{6}{|l|}{$\begin{array}{l}\text { AXES DE GESTION } \\
\text { DU PERSONNEL }\end{array}$} \\
\hline Orientation politique & n.s. ${ }^{b}$ & n.s. & n.s. & $3,05^{c}$ & $4,07 \%$ \\
\hline $\begin{array}{l}\text { Pourcentage } \\
\text { du personnel }\end{array}$ & $\begin{array}{l}(0,04) \\
\text { n.s. }\end{array}$ & $(0,04)$ & & & \\
\hline $\begin{array}{l}\text { Orientation administrative } \\
\text { du personnel }\end{array}$ & $(0,06)$ & $2,77^{d}$ & $\begin{array}{c}\text { n.s. } \\
(0,06)\end{array}$ & n.s. & $3,89 \%$ \\
\hline
\end{tabular}

\section{POIDS ACCORDÉ}

\section{PAR LE DIRIGEANT}

\begin{tabular}{lccccc}
$\begin{array}{l}\text { Gestion du } \\
\text { personnel }\end{array}$ & n.s. & 2,41 & n.s. & n.s. & $\begin{array}{c}2,39 \% \\
\text { Management }\end{array}$ \\
$\begin{array}{l}\text { Mes hommes } \\
\text { des }\end{array}$ & n.s. & 5,33 & 2.19 & n.s. & $3,09 \%$ \\
\hline
\end{tabular}

$a$ Coefficient de détermination ( $R 2)$ et significativité globale $(\operatorname{Pr}>\mathrm{F})$ de l'équation d'analyse de variance.

b n.s.: l'effet du facteur est non significatif (le seuil de signification est supérieur à $10 \%$ ).

c Valeur $\mathrm{F}$ du test de Fisher et probabilité critique $(\operatorname{Pr}>\mathrm{F})$ pour chaque facteur dans le modèle d'analyse.

$d$ L'effet d'interaction entre la fréquence à l'exportation et l'expérience internationale de l'entreprise est significatif au seuil de $12 \%$.

$\mathrm{Si}$, de manière globale, le développement international des PMI de notre échantillon semble avoir peu de répercussions sur les pratiques de gestion du personnel, les seuls effets significatifs s'interprètent comme suit :

- plus les moyens humains affectés à l'activité export, en particulier la présence d'un spécialiste en commerce international, sont réguliers et l'organisation export structurée, et plus les politiques du personnel ont une dimension stratégique (gestion des compétences, prévision, organisation du travail, etc.). Ce résultat est le reflet d'une volonté stratégique de développement réel sur les marchés étrangers par l'intermédiaire d'une organisation à l'export axée sur la présence d'un personnel spécialisé, voire d'un service export (Léo, Monnoyer et Philippe, 1990 ; Samiee et Walters, 1990);

- plus les exportations sont régulières au cours des cinq dernières années qui précèdent 1990, voire une expérience internationale élevée (première exportation antérieure à 1974), plus la dimension administrative est « enrichie » par une attention particulière à la gestion des salaires couplée à des politiques 
blement, de mieux contrôler les frais structurels. Ce résultat ne contredit pas la perception qu'ont les dirigeants de PME-PMI de la concurrence, qui s'exerce surtout par les prix sur les marchés étrangers et constitue le principal frein à l'exportation (Soum et al., 1991; Koe et Robicheaux, 1988).

Les effets du degré de développement international sur le poids accordé par le dirigeant à la gestion et au management du personnel ne sont pas plus nets. Les seuls véritablement significatifs sont la fréquence à l'exportation et, à un niveau plus faible, le risque à l'exportation (tableau 1).

- En effet, on peut remarquer que seule la fréquence à l'export agit de manière très significative $(\mathrm{p}<0,01)$ sur l'importance accordée par le dirigeant à la direction et à l'organisation des hommes. Ainsi, plus la politique d'exportation est régulière au cours des cinq dernières années, plus le dirigeant accorde une place importante au management des hommes.

- Dans une moindre mesure, le poids accordé au management des hommes est aussi influencé par le risque à l'export $(\mathrm{p}<0,05)$. Plus la zone d'intervention commerciale à l'export est importante et les marchés étrangers éloignés, plus, dans ce cas de figure aussi, le dirigeant de PMI accordera d'importance à la dimension direction et organisation du personnel de son entreprise.

En fin de compte, la réponse que l'on peut donner à la question Q1 va dans le sens d'une faiblesse des effets du développement international tant sur les principaux axes des pratiques de gestion du personnel que sur le poids accordé à la gestion et au management du personnel ; car cinq effets seulement sur les seize testés (non compris les effets d'interaction) sont significatifs au seuil de probabilité de $10 \%$ et trois, seulement, pour un risque de première espèce de $5 \%$ (tableau 1). Autrement dit, il ne semble pas y avoir d'effets lourds de l'international sur la GRH de la PME.

Cependant, les effets significatifs les plus nombreux (trois sur cinq) sont ceux associés à la fréquence d'exportation. Ce résultat semble indiquer que la relation entre gestion du personnel et internationalisation de l'entreprise est plus saisissable à la lumière des différentes phases ou étapes de son processus de développement international.

\subsection{Effets des caractéristiques de la PMI sur la GRH}

La mise en relation des caractéristiques de base de la PMI que nous avons retenue, avec d'une part, les principaux axes de gestion du personnel et, d'autre part, le poids accordé par le dirigeant à la gestion et au management du personnel, fournit les résultats consignés au tableau 2. 
TABLEAU 2

Effets des caractéristiques de la PMI sur la GRH

\begin{tabular}{|c|c|c|c|c|}
\hline & \multicolumn{4}{|c|}{ CARACTÉRISTIQUES DE LA PMI } \\
\hline & $\begin{array}{l}\text { Taille de } \\
\text { l'entreprise }\end{array}$ & $\begin{array}{l}\text { Maturité de } \\
\text { l'entreprise }\end{array}$ & $\begin{array}{l}\text { Secteur d'activités } \\
\text { de l'entreprise }\end{array}$ & $\begin{array}{c}R 2 \\
(\operatorname{Pr}>\text { F })\end{array}$ \\
\hline \multicolumn{5}{|l|}{$\begin{array}{l}\text { AXES DE GESTION } \\
\text { DU PERSONNEL }\end{array}$} \\
\hline $\begin{array}{l}\text { Orientation politique } \\
\text { du personnel }\end{array}$ & $\begin{array}{c}3,17 \\
(0,01)\end{array}$ & $\begin{array}{c}4,47 \\
(0,00)\end{array}$ & n.s. & $\begin{array}{l}10,72 \% \\
(0,00)\end{array}$ \\
\hline $\begin{array}{l}\text { Orientation administrative } \\
\text { du personnel }\end{array}$ & $\begin{array}{c}5,41 \\
(0,00)\end{array}$ & n.s. & n.s. & $\begin{array}{l}8,66 \% \\
(0,00)\end{array}$ \\
\hline \multicolumn{5}{|l|}{$\begin{array}{l}\text { POIDS ACCORDÉ } \\
\text { PAR LE DIRIGEANT }\end{array}$} \\
\hline $\begin{array}{l}\text { Gestion } \\
\text { du personnel }\end{array}$ & $\begin{array}{c}2,22 \\
(0,05)\end{array}$ & n.s. & n.s. & $\begin{array}{l}3,82 \% \\
(0,05)\end{array}$ \\
\hline $\begin{array}{l}\text { Management } \\
\text { des hommes }\end{array}$ & $\begin{array}{c}3,03 \\
(0,01)\end{array}$ & n.s. & n.s. & $\begin{array}{l}5,16 \% \\
(0,01)\end{array}$ \\
\hline
\end{tabular}

Pour ce qui est de l'impact des caractéristiques de la PMI sur les axes de gestion du personnel, nous observons les effets significatifs suivants :

- plus la taille de l'entreprise ( $\mathrm{p}<0,01)$ et son degré de maturité $(\mathrm{p}<0,00)$ sont élevés, plus les pratiques de gestion du personnel ont une dimension stratégique associée, entre autres, à la gestion des compétences (emploi, formation) et à la gestion prévisionnelle du personnel. Par ailleurs, on peut constater que la taille et la maturité expliquent $10,72 \%$ de la variance totale de l'orientation politique du personnel («réactive versus proactive»).

- plus la taille de l'entreprise $(p<0,01)$ est importante, plus celle-ci accorde d'importance aux aspects administratifs de la gestion du personnel et à la gestion des rémunérations et de l'emploi. Même si la maturité et le secteur d'activités de l'entreprise n'interviennent pas de manière significative, la taille explique à elle seule près de $9 \%$ de la variance totale de l'orientation administrative du personnel («contrainte versus enrichie»).

En ce qui concerne l'impact des caractéristiques de la PMI sur le poids accordé par le dirigeant à la gestion et au management du personnel, la taille est une fois encore présente. Outre le fait qu'il soit ici unique, l'effet de la taille est très fortement significatif $(\mathrm{p}<0,01)$ sur l'importance accordée par le dirigeant de PMI à la direction et à l'organisation des ressources humaines. Ainsi, plus la taille de l'entreprise s'élève, plus l'importance accordée aux hommes par le dirigeant a tendance à s'accroître significativement. Beaucoup moins significative est l'incidence 
de la taille sur le degré d'importance accordée par le dirigeant à la gestion du personnel $(p<0,05)$.

En résumé, la réponse à la question $\mathrm{Q} 2$ fait ressortir de manière très nette les incidences de la taille de l'entreprise tant sur les pratiques de GRH que sur le poids accordé par le dirigeant de PMI à la gestion et au management de son personnel. Ce résultat ne fait que confirmer l'importance des paramètres structurels dans la compréhension des orientations retenues, en matière de GRH, par la PME (Mahé de Boislandelle, 1988 ; Benoît et Rousseau, 1990 ; Bayad, 1991). Cela est d'autant plus important que la taille exerce une influence tout aussi significative sur le comportement commercial de la PMI exportatrice française (Reffait et Roux, 1981).

\subsection{Les autres résultats}

- Du même ordre de grandeur que pour les effets des caractéristiques de la PMI, et comme le présente le tableau 3, le poids accordé par le dirigeant à la gestion et au management des hommes exerce une influence très significative sur les principales dimensions retenues des pratiques de GRH des PME industrielles de notre échantillon.

TABLEAU 3

Effets du poids accordé par le dirigeant aux ressources humaines sur les axes de gestion du personnel

\begin{tabular}{lccc}
\hline & \multicolumn{2}{c}{ POIDS ACCORDÉ PAR LE DIRIGEANT } \\
& $\begin{array}{c}\text { Gestion } \\
\text { personnel }\end{array}$ & $\begin{array}{c}\text { Management } \\
\text { hommes }\end{array}$ & $\begin{array}{c}R 2 \\
(P r>F)\end{array}$ \\
\hline AXES DE GESTION & & & \\
DU PERSONNEL & & 4,04 & \\
$\begin{array}{l}\text { Orientation politique } \\
\text { du personnel }\end{array}$ & 6,18 & $(0,01)$ & $9,93 \%$ \\
$\begin{array}{l}\text { Orientation administrative } \\
\text { du personnel }\end{array}$ & $(0,00)$ & n.s. & $(0,00)$ \\
\hline & $(0,00)$ & & $4,83 \%$ \\
\hline
\end{tabular}

Pour répondre à la question $\mathrm{Q} 3$, on constate que plus le poids accordé à la gestion $(p<0,00)$ et au management $(p<0,01)$ des hommes par le dirigeant est important, plus la politique du personnel prend une dimension stratégique avec un accent sur les principales pratiques de GRH déjà soulignées (emploi, formation, prévision, organisation du travail). Le pourcentage de variance expliquée est, là aussi, non négligeable puisqu'il s'élève à près de $10 \%$. Par contre, seule l'importance accordée à la gestion du personnel par le dirigeant est significative $(\mathrm{p}<0,00)$ en ce qui concerne l'effet sur la dimension administrative de gestion du personnel. 
Ce dernier résultat, bien qu'associé à notre échantillon de PMI lorraines, est révélateur de la dimension administrative généralement attachée à la gestion du personnel dans une PME. On ne peut aussi que remarquer indirectement le rôle joué par le dirigeant de PME en matière de GRH (Mahé de Boislandelle, 1988; Bayad et Nebenhaus, 1996). En effet, on constate une cohérence entre le poids que le dirigeant de PMI accorde à ses ressources humaines et les pratiques déclarées de gestion du personnel de son entreprise.

- En ce qui concerne les questions Q4 et Q5, relatives aux effets des principales orientations de GRH et du poids accordé par le dirigeant à la gestion et au management du personnel sur la performance à l'exportation, les résultats (tableau 4) indiquent une absence quasi générale d'incidences significatives.

En effet, excepté l'impact, à la limite de la significativité $(p<0,08)$, du degré d'importance accordé par le dirigeant à la direction et à l'organisation des hommes, les autres influences testées ne révèlent rien de statistiquement significatif sur la performance à l'exportation dans notre échantillon de travail.

TABleau 4

Effets des orientations de GRH sur la performance à l'exportation

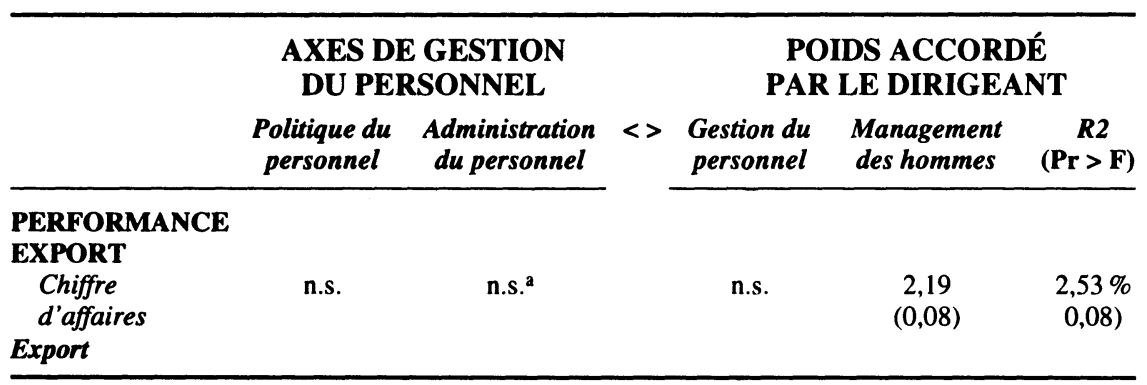

a L'axe administration du personnel exerce un effet significatif sur le chiffre d'affaires export au seuil de $11,5 \%$.

\section{Conclusions et implications}

En reprenant de manière synthétique les réponses aux questions constitutives de notre problématique, il est possible de présenter quelques remarques et implications associées aux résultats obtenus.

Tout d'abord, il semble que le degré de développement international de la PMI n'exerce pas d'effets lourds tant sur les pratiques de GRH que sur l'importance accordée par le dirigeant à la gestion et au management de son personnel. Ce résultat est conforme à celui obtenu par Hermel (1991) auprès de grandes entreprises ; 
$65 \%$ des entreprises interrogées par cet auteur considèrent que l'international n'a qu'un effet indirect sur la GRH. Cependant, comme nous l'avons souligné, les effets significatifs associés en particulier à la fréquence d'exportation semble aller dans le sens d'une approche de la GRH de la PMI selon la phase du processus d'exportation dans laquelle elle se situe (Yaprak, 1985 ; Vozikis et Mescon, 1985).

- Ainsi, il apparaît en premier lieu que le dirigeant de PME doit accorder une attention particulière à la gestion de ses ressources humaines dès lors qu'il s'oriente vers un engagement plus fort sur les marchés étrangers. Cette attention est relative tant aux aspects stratégiques liés à la gestion et à l'organisation des compétences qu'aux aspects administratifs liés à la gestion des salaires et aux politiques de rémunération face à un engagement international de plus en plus volontaire. Cette implication est d'autant plus nette que le degré d'engagement à l'export semble exercer une certaine influence sur le poids accordé par le dirigeant à la dimension management des ressources humaines.

- En second lieu, il convient de rappeler au dirigeant de PMI non exportatrice que certaines variables de GRH comme la gestion de l'emploi (recrutement, mobilité, etc.), la gestion des compétences et la gestion des rémunérations sont des paramètres sensibles face à l'international et qu'une attention plus importante doit leur être accordée. Comme le souligne Hermel (1991), ces variables «sont porteuses d'un potentiel d'intégration par delà les frontières [...] C'est à travers de telles variables que se joue le degré de fragilité ou de solidité du tissage international de toute organisation ». Par ailleurs, la gestion des ressources humaines dans le contexte de l'espace social européen de 1993 révèle tout autant de "contingences que de convergence », en matière de conditions d'emploi et de rémunération, qui nécessitent de la part des dirigeants des modes d'action et de réflexion de GRH à l'échelle européenne.

Ensuite, il apparaît que parmi les caractéristiques de la PMI, la taille exerce une incidence très significative sur les principales orientations de gestion du personnel, mais aussi sur le poids accordé par le dirigeant aux ressources humaines. Ce résultat va dans le sens d'une intégration stratégique des ressources humaines au développement de l'entreprise (Besseyre Des Horts, 1991) avec l'accroissement de la taille. Il faut cependant rappeler que la taille de l'entreprise n'est pas indépendante de son stade de développement et de la technologie utilisée (Bayad, 1991). Cela souligne le rôle contingent de la taille sur les choix organisationnels de l'entreprise et soulève ainsi la question de la prépondérance du structurel sur le stratégique, en matière de GRH, dans une PME. 


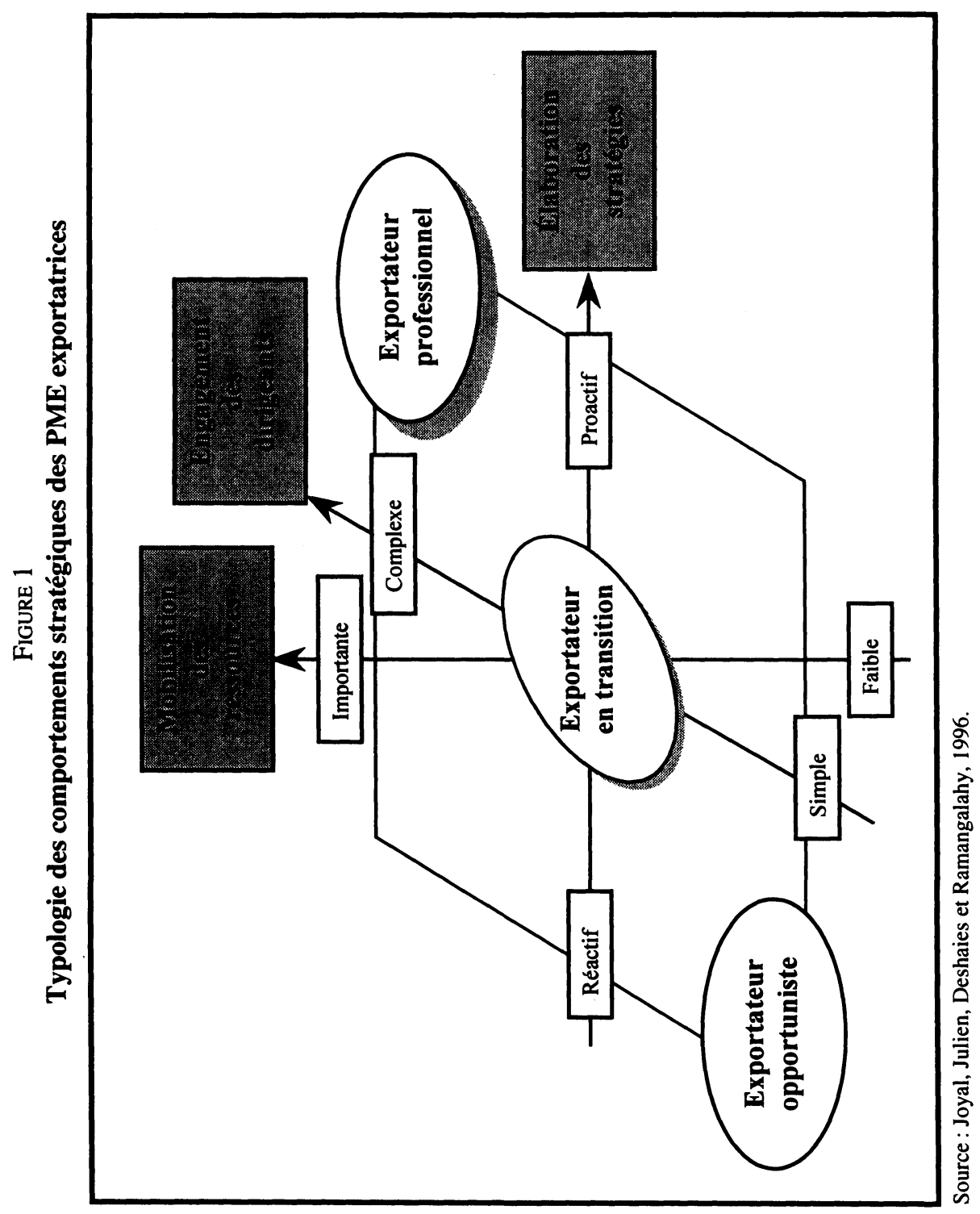

Enfin, l'absence d'effets des principales orientations de gestion du personnel sur la performance à l'export de la PMI témoigne de l'importance des autres dimensions structurelles et stratégiques dans l'engagement international (Léo, Monnoyer-Longe et Philippe, 1990). En particulier, il semble qu'il faille rechercher les facteurs de performance à l'exportation dans le choix des formes organisationnelles 
susceptibles de mieux répondre aux difficultés ou aux opportunités de l'engagement international. Ainsi, les effets de la GRH sur la performance à l'export devraient donc mieux se comprendre en tenant compte des formes organisationnelles mises en place pour répondre aux défis de l'internationalisation.

De ce point de vue, la typologie des PME exportatrices de Joyal, Julien, Deshaies et Ramangahaly (1996) pourrait servir de grille d'approche des comportements organisationnels et stratégiques à l'international des PME.

Cela est d'autant plus intéressant que, pour les auteurs, «le développement des exportations implique des mesures de support dans les autres fonctions de l'entreprise : la modernisation de la production, le développement des ressources humaines, la stabilisation des activités de recherche-développement et la pratique d'une veille commerciale, technologique et concurrentielle touchant les marchés ciblés ». Dans cette perspective, une piste de recherche pourrait consister dans le développement d'une lecture des activités de GRH dans un tel cadre conceptuel.

\section{Conclusion}

En conclusion et tenant compte des limites inhérentes à cette analyse, en particulier le côté statique de l'approche de l'engagement sur les marchés étrangers, il semble que l'effet de l'international se fera sentir dans la gestion des compétences, surtout au regard des besoins spécifiques de l'organisation export.

\section{Bibliographie}

AABY, N.-E. et S.F. SLATER (1989), « Management influences on export performance : A review of the empirical literature 1978-88", International Marketing Review, vol. $6, \mathrm{n}^{\circ} 4$, p. 7-26.

ADLER, N.J. (1994), Comportement organisationnel. Une approche multiculturelle, Collection «Perspective internationale», Montréal, Éditions Reynald Goulet inc., 324 p.

Audibet, J. et C. PARLAnt (1990), Gestion du personnel expatrié, Paris, Nouvelles Éditions Fiduciaires, Levallois Perret, 223 p.

BAIRD, L. et L. MESHOULAM (1988), « Managing two fits of strategic human resource management », Academy of Management Review, 13, p. 116-128.

BAIRD, I.S., M.A. LYLES et J.B. ORRIS (1994), «The choice of international strategy by small businesses », Journal of Small Business Management, vol. 32, $\mathrm{n}^{0}$ 1, p. 48-59. 
BARTOLI, A. (1991), «Stratégie et ressources humaines : Analyses cliniques, 410-419», Actes du $2^{e}$ Congrès AGRH, Pour une vision de la GRH, ESSEC, Cergy, 14-15 novembre, $635 \mathrm{p}$.

BAYAD, M. (1991), «La gestion des effectifs dans les PME industrielles: Quelques éléments de réflexion, 347-357 », Actes du $2^{e}$ Congrès AGRH, Pour une vision de la GRH, ESSEC, Cergy, 14-15 novembre, $635 \mathrm{p}$.

BAYAD, M. et D. Nebenhaus (1996), «Préoccupations de GRH et profil des dirigeants de PME », Économies et sociétés, série «Sciences de gestion», n 22, p. 185-203.

BAyAd, M. et J.L. HerRmANn (1992), Panel des PMI lorraines : Rapport technique de la troisième vague, Enquête 1991, Centre régional de documentation en gestion, Nancy, juin, $397 \mathrm{p}$.

BENOÎT, C. et M.D. Rousseau (1990), «La gestion des ressources humaines dans les PME au Québec », Revue Internationale PME, vol. 3, n 1, p. 39-55.

BESSEYRE DES HORTS, C.H. (1988), Vers une gestion stratégique des ressources humaines, Paris, Éd. d'Organisation, 224 p.

BESSEYRE DES HORTS, C.H. (1991), «La gestion internationale des carrières dans le contexte européen », Revue Française de Gestion, vol. 83, p. 84-88.

Bumolt, T.H.A. et P.S. ZWART (1994), «The impact of internal factors on the export success of Dutch small and medium-sized firms ", Journal of Small Business Management, vol. 32, $\mathrm{n}^{\circ}$ 2, p. 69-83.

BILKEY, W.J. et G. TESAR (1977), «The export behavior of smaller-sized Wisconsin manufacturing firms ", Journal of International Business Studies, vol. 8, p. 93-98.

BILKEY, W.J. (1978), "An attempted integration of the literature on export behavior of firms », Journal of International Business Studies, vol. 9, p. 33-46.

BouRnOIS, F. (1991), «Pratiques de gestion des ressources humaines en Europe : Données comparées ", Revue Française de Gestion, vol. 83, p. 68-83.

CAvusGiL, S.T. (1984), "Differences among exporting firms based on their degree of internationalization ", Journal of business research, vol. 12, $\mathrm{n}^{\circ} 3$, p. 195-208.

CAVUSGIL, S.T. et J.R. NEVIN (1981), «State of the art in international marketing ", dans B.M. Enis et K.J. Roaring (dir.), Review of Marketing, American Marketing Association, Chicago, p. 196-216.

CAvusgil, S.T. et J.R. NEvin (1981), «Internal determinants of export marketing behavior : an empirical investigation ", Journal of Marketing Research, février, vol. 18, p. 114-119.

Chaganti, R., R. Chaganti et V. Mahajan (1989), « Profitable small business strategies under different types of competition», Entrepreneurship, Theory and Practice, vol. 13, no 3, printemps, p. 21-35. 
CHANDLER, A.D. (1986), « The evolution of modern global competition », dans M.E. Porter (dir.), Competition in Global Industries, Boston, Harvard Business School Press, p. $405-448$.

CHASE, H.C. (1984), « Export expansion and small business productivity », American Journal of Small Business, vol. 8, $\mathrm{n}^{\circ} 4$, p. 21-27.

CZINKOTA, M.R. et M. URSIC (1991), «Classification of exporting firms according to sales and growth into a share matrix », Journal of Business Research, vol. 22, p. 243-253.

DE LEERSNYDER, J.M. (1980), «Beaucoup de PMI ne sont pas faites pour exporter », Le Monde de l'Économie, 18 mars, p. 25.

D'IRIBARNE, P. (1991), «Les entreprises françaises dans une Europe multiculturelle», Revue Française de Gestion, vol. 83, p. 98-103.

DiTCHL, E. et al., (1984), "The export-decision of small and medium-sized firms : a review », Management International Review, vol. 24, p. 49-61.

Dowling, P.J. et R.S. SCHULER (1990), International Dimensions of Human Resource Management, Boston, MA, PWS-Kent Publishing, 192 p.

Fombrun C.J., M.A. Devanna et N.M. Tichy (dir.) [1984], Strategic Human Resource Management, New-York, J. Wiley.

GARAND, D. et B. FABI (1991), «Fondements des pratiques de GRH en PME : Formalisation, vision entrepreneuriale et modèle contingentiel, 324-336», Actes du $2^{e}$ Congrès AGRH, Pour une vision de la GRH, ESSEC, Cergy, 14-15 novembre, 635 p.

GOMEZ-MEIJA, L.R. (1988), « The role of human resources strategy in export performance : a longitudinal study », Strategic Management Journal, vol. 9, p. 493-505.

Hermel, P. (1991), «Développement international et management des ressources humaines, 373-381 », Actes du $2^{e}$ Congrès AGRH, Pour une vision de la GRH, ESSEC, Cergy, 14-15 novembre, 635 p.

HERMEL, P. (1993), Management européen et international, Paris, Economica gestion.

JOFFRE, P. (1994), Comprendre la mondialisation de l'entreprise, Paris, Economica, Collection «Gestion poche», $111 \mathrm{p}$.

JOHANNISSON, B. (1994), «Building a 'global' strategy. Internationalizing small firms through local networking », dans J.J. Obrecht et M. Bayad (dir.), Les PME/PMI et leur contribution au développement régional et international, Actes de la $39^{\mathrm{e}}$ Conférence annuelle mondiale de l'International Council for Small Business (ICSB), Strasbourg, 27-29 juin, p. A 127-135.

Joyal, A., P.-A. Julien, L. Deshaies et C. Ramangalahy (1996), «Une typologie des comportements stratégiques des PME exportatrices », Gestion, vol. 21, n 1, p. 29-37.

Koe, A.C. et R.A. Robicheaux (1988), "Variations in export performance due to differences in export marketing strategy : implications for industrial marketers », Journal of Business Research, vol. 17, p. 249-258. 
LÉo, P.Y., M.C. MonNoyer-Longe et J. PhILIPPE (1990), PME : Stratégies internationales, Paris, Economica, 266 p.

LÉo, P.Y. (1993), "Le développement international des PME / PMI : outils pour une analyse stratégique ", Communication au Colloque PME/PMI, Développement international, CER/GREPME, Aix-en-Provence, juin.

MAHÉ DE BoISLANDELlE, H. (1988), Gestion des ressources humaines dans les PME, Paris, Economica, $322 \mathrm{p}$.

MALEKZADEH, A.R. et A. NAHAVANDI (1985), «Small business exporting : misconceptions are abondant », American Journal of Small Business, vol. 9, n 4, p. 7-14.

MIESENBOCK, K.J. (1988), «Small businesses and exporting : a literature review », International Small Business Management, vol. 6, $\mathrm{n}^{\circ} 2$, p. 42-61.

NAMIKI, N. (1988), «Export strategy for small business », Journal of Small Business Management, vol. 26, $\mathrm{n}^{\circ}$ 2, p. 33-37.

OGBueHI, A.O. et T.A. LoNGFELlow (1994), «Perceptions of U.S. manufacturing SMEs concerning exporting : a comparison based on export experience », Journal of Small Business Management, vol. 32, $\mathrm{n}^{\circ}$ 4, octobre, p. 37-47.

OHмaE, K. (1985), Triad Power : The Coming Shape of Global Competition, New York, Free Press.

ORSINI, J. (1985), «Les PME face aux nouvelles conditions de l'échange international », Cahiers de recherche de l'IAE de Lille, vol. 85, nº 8, 14 p.

Peretti, J.M., D. CAZAL et F. QuiQuANDON (1990), Vers le management international des ressources humaines, Paris, Éditions Liaisons, 283 p.

Perlmutter, H.V. et D.A. HeEnAN (1979), Multinational Organization Development : A Social Architectural Perspective, Reading, MA, Addison-Wesley.

PORTER, M.E. (1986), Competition in Global Industries, Boston, Harvard Business School Press.

Reffait, P. et E. Roux (1981), «Le profil idéal de la PMI exportatrice», Revue Française de Gestion, janvier-février, p. 88-96.

Roux, E. (1986), «Les modèles intégrés de la décision d'exporter en PME/PMI», Recherche et Applications en Marketing, vol. 3, p. 27-42.

Roux, E. (1991), Les facteurs explicatifs de la décision d'exporter en PMI: Rôle de l'attitude du dirigeant envers le risque, thèse de doctorat d'État des sciences de gestion, IAE, Aix-Marseille.

SAMIEE, S. et G.P. WALTERS (1990), «Influence of firm size on export planning and performance », Journal of Business Research, vol. 20, p. 235-248.

SAPORTA, B. (1986), «Les PME-PMI face au développement international », Cahiers de recherche, $52 \mathrm{p}$. 
SCHUler, R.S., P.J. Dowling et H. DE CIERI (1993), "An integrative framework of strategic international human resource management », International Journal of Human Resource Management, vol. 4, nº 4, décembre, p. 717-764.

Soum, J.C. et al. (1991), "Des PME très entreprenantes ", Le Moci, hebdo., no 999, 18 novembre, 15-21.

Thevenet, M. (1991), «Une gestion des ressources humaines européenne est-elle possible? ", Revue Française de Gestion, vol. 83, p. 62-67.

Urban, S. (1993), Management International, Paris, Litec, Collection « Les essentiels de la gestion ", $205 \mathrm{p}$.

VIDAL, Y. (1991), Cadres sans frontières, Paris, ESF Éditeur, 174 p.

VozIKIS, G.S. et T.S. MESCON (1985), «Small exporters and stages of development : an empirical study », American Journal of Small Business, p. 49-64.

WRIGHT, P.C. (1993), «The personal and the personnel adjustments and costs to small businesses entering the international market place», Journal of Small Business Management, vol. 31, no 1, janvier, p. 83-93.

YAPRAK, A. (1985), "A empirical study of the differences between small exporting and non-exporting US firms », International Marketing Review, p. 72-83. 